University of Windsor

Scholarship at UWindsor

1976

\title{
Calculated anisotropies for the quenching radiation in the hydrogen isoelectronic sequence
}

\author{
Gordon W. F. Drake
}

University of Windsor

C. P. Lin

Follow this and additional works at: https://scholar.uwindsor.ca/physicspub

Part of the Physics Commons

\section{Recommended Citation}

Drake, Gordon W. F. and Lin, C. P.. (1976). Calculated anisotropies for the quenching radiation in the hydrogen isoelectronic sequence. Physical Review A, 14 (3), 1296-1298.

https://scholar.uwindsor.ca/physicspub/105

This Article is brought to you for free and open access by the Department of Physics at Scholarship at UWindsor. It has been accepted for inclusion in Physics Publications by an authorized administrator of Scholarship at UWindsor. For more information, please contact scholarship@uwindsor.ca. 


\title{
Calculated anisotropies for the quenching radiation in the hydrogen isoelectronic sequence*
}

\author{
G. W. F. Drake ${ }^{\dagger}$ and Chien-ping Lin \\ Department of Physics, University of Windsor, Windsor, Ontario, Canada N9B 3P4 \\ (Received 3 May 1976)
}

\begin{abstract}
Accurate values of the Lamb shift can be derived from measurements of the anisotropy in the angular distribution of the radiation accompanying the electric field induced $2 s-1 s$ transition of hydrogen. Calculations of the anisotropy, including relativistic and hyperfine structure corrections, are presented for the hydrogenic ions up to $\mathrm{S}^{15+}$. The data enable the calculation of the Lamb shift from a measured value of the anisotropy.
\end{abstract}

\section{INTRODUCTION}

There has been much recent interest, both experimental $^{1,2}$ and theoretical, ${ }^{3,4}$ in the Lamb shifts of heavy hydrogenic systems. In previous papers, ${ }^{5,6}$ we have suggested that measurements of the angular anisotropy in the electric field quenching radiation from the metastable $2 s_{1 / 2}$ state may provide an accurate method of deriving the Lamb shifts of hydrogenic ions. A nonperturbative theory of the process has been developed ${ }^{7}$ and the technique has been tested to high precision in hy drogen and deuterium. ${ }^{8}$

For hydrogenic ions with nuclear charge $Z$ greater than five or six, first-order perturbation theory is adequate for readily obtainable electric quenching fields (i.e., fields up to about $50 Z^{4} \mathrm{~V} /$ $\mathrm{cm}$ ). Under these conditions, the anisotropy is very nearly equal to its zero-field value, and therefore independent of field strength. In this paper, we present calculations of the zero-field anisotropies as a function of the Lamb shift for the ions up to hydrogenic sulphur. Lamb shifts can then be derived when experimental measurements of the anisotropy are available. The calculations include nuclear-spin and relativistic effects.

\section{THEORY}

The theory presented previously ${ }^{7,8}$ is modified here to include relativistic corrections in the evaluation of the transition matrix elements. The calculations are performed in the coupled representation $\vec{F}=\vec{j}+\vec{I}$, where $\vec{j}$ is the total electronic angular momentum and $\overrightarrow{\mathrm{I}}$ is the nuclear spin. We assume that the metastable ion beam is initially an incoherent mixture of all the hyperfine states of the $2 s_{1 / 2}$ level with equal statistical weights. Then in the limit of weak-field strength $\mathcal{F}$, the induced electric dipole transition rate can be written in the form

$$
\begin{aligned}
I(\hat{e})= & \frac{2 \pi}{\hbar} \frac{\rho(\bar{\omega})}{(2 I+1)(2 j+1)} \\
& \times\left(|\hat{e} \cdot \hat{F}|^{2}|A|^{2}+|\hat{e} \times \hat{F}|^{2}\left|A^{\prime}\right|^{2}\right),
\end{aligned}
$$

where $\rho(\bar{\omega})$ is the density of photon states at the average emitted frequency $\bar{\omega}, \hat{e}$ is the photon polarization vector, and $\hat{F}$ is a unit vector pointing in the electric field direction. The coefficients $A$ and $A^{\prime}$ correspond to transitions in which $\Delta M=0$ and \pm 1 , respectively. $|A|^{2}$ is given by (in atomic units)

$$
\begin{array}{r}
|A|^{2}=\sum_{F, M, F^{\prime}} \mid \sum_{j^{\prime \prime} F^{\prime \prime}}\left\langle 1 s, F^{\prime} M^{\prime}|\vec{\alpha} \cdot \vec{\alpha}| 2 p, j^{\prime \prime} F^{\prime \prime} M\right\rangle \\
\times\left.\frac{\left\langle 2 p, j^{\prime \prime} F^{\prime \prime} M|\mathcal{F} z| 2 s, F M\right\rangle}{E\left(2 p, j^{\prime \prime} F^{\prime \prime}\right)-E(2 s, F)}\right|^{2}
\end{array}
$$

with $M^{\prime}=M$. Here, $\vec{\alpha}$ is the $4 \times 4$ Dirac matrix and $\vec{a}$ is the electric dipole vector potential for the emitted photon. The corresponding expression for $\left|A^{\prime}\right|^{2}$ is the same except that $M^{\prime}=M \pm 1$.

For electric dipole transitions, the relativistic matrix elements in Eq. (2) are both of the form $\left\langle\gamma^{\prime} j^{\prime} I F^{\prime} M^{\prime}|T(1 q)| \gamma j I F M\right\rangle$, where $T(1)$ is an irreducible tensor of rank 1 . Using standard vector coupling techniques, the matrix elements can be written in terms of $3-j$ and $6-j$ symbols as

$$
\begin{aligned}
\left\langle\gamma^{\prime} j^{\prime} I F^{\prime} M^{\prime}|T(1 q)| \gamma j I F M\right\rangle & (-1)^{F^{\prime}-M^{\prime}}\left(\begin{array}{ccc}
F^{\prime} & 1 & F \\
-M^{\prime} & q & M
\end{array}\right) \\
& \times(-1)^{j^{\prime}+I+F+1}\left[(2 F+1)\left(2 F^{\prime}+1\right)\right]^{1 / 2} \\
& \times\left\{\begin{array}{ccc}
j^{\prime} & F^{\prime} & I \\
F & j & 1
\end{array}\right\}\left\langle\gamma^{\prime} j^{\prime}\|T(1)\| \gamma j\right\rangle . \quad(3)
\end{aligned}
$$

Following Grant, ${ }^{9}$ the reduced matrix elements are

1296 


$$
\begin{aligned}
\left\langle\gamma^{\prime} j^{\prime}\|T(1)\| \gamma j\right\rangle= & (-1)^{j^{\prime}-1 / 2}\left[\left(2 j^{\prime}+1\right)(2 j+1)\right]^{1 / 2} \\
& \times\left(\begin{array}{ccc}
j^{\prime} & 1 & j \\
\frac{1}{2} & 0 & -\frac{1}{2}
\end{array}\right) \bar{M}_{j^{\prime} j},
\end{aligned}
$$

where in the Coulomb gauge

$$
\begin{aligned}
\bar{M}_{j, j}=i(\omega / \pi c)^{1 / 2}\left\{(2)^{-1 / 2}\left[\left(\kappa^{\prime}-\kappa\right) I_{2}^{+}+2 I_{2}^{-}\right]\right. \\
\left.-(2)^{1 / 2}\left[\left(\kappa^{\prime}-\kappa\right) I_{0}^{+}-I_{0}^{-}\right]\right\}
\end{aligned}
$$

for the $\vec{\alpha} \cdot \vec{Q}$ matrix element, and

$$
\bar{M}_{j^{\prime} j}=\mathfrak{F} \int_{0}^{\infty}\left(P^{\prime} P+Q^{\prime} Q\right) r d r
$$

for the $\mathfrak{F} z$ matrix element. In the above,

$$
I_{L}^{ \pm}=\int_{0}^{\infty}\left(P^{\prime} Q \pm Q^{\prime} P\right) j_{L}(\omega r / c) d r
$$

$j_{L}(x)$ is the spherical Bessel function, and $P$ and $Q$ are the large and small components of the relativistic radial wave functions. ${ }^{10}$ The integrals $I_{L}^{ \pm}$ were calculated analytically by expanding $j_{L}(\omega r / c)$ and retaining all terms up to $(\omega r / c)^{2}$.

The quantity of interest in anisotropy measurements is the total intensity summed over both polarizations emitted in directions parallel and perpendicular to the electric field. Summing Eq. (1) over two perpendicular vectors $\hat{e}$, both perpendicular to the direction of propagation, yields

$$
\begin{aligned}
I(\theta, \varphi)=\frac{2 \pi}{\hbar} \frac{\rho(\bar{\omega})}{(2 I+1)(2 j+1)}[ & {\left[|A|^{2}\left(1-\cos ^{2} \theta\right)\right.} \\
& \left.+\left|A^{\prime}\right|^{2}\left(1+\cos ^{2} \theta\right)\right],
\end{aligned}
$$

where $\theta$ and $\varphi$ are the spherical angles defining the direction of propagation and $\hat{F}$ is taken to point in the $z$ direction. ${ }^{11}$ The anisotropy $R$ is defined to be

$$
R=\left(I_{11}-I_{\perp}\right) /\left(I_{11}+I_{\perp}\right),
$$

where $I_{11}=I(0,0)$ and $I_{\perp}=I\left(\frac{1}{2} \pi, 0\right)$. Thus from Eq. (8)

$$
R=\left(\left|A^{\prime}\right|^{2}-|A|^{2}\right) /\left(3\left|A^{\prime}\right|^{2}+|A|^{2}\right) \text {. }
$$

The calculated values of $R$ are presented in the following section.

\section{RESULTS}

In addition to the relativistic matrix elements described in the preceding section, we also need the energy differences in the denominator of Eq. (2), including hyperfine structure. The calculated anisotropies are parametrized in terms of the Lamb shift $S=E\left(2 s_{1 / 2}\right)-E\left(2 p_{1 / 2}\right)$ uncorrected for hyperfine structure. The fine-structure splitting $E\left(2 p_{3 / 2}\right)-E\left(2 p_{1 / 2}\right)$, which to a first approximation is a non-QED effect, can be estimated to sufficient accuracy from the formula ${ }^{12}$

$$
\begin{aligned}
E\left(2 p_{3 / 2}\right)-E\left(2 p_{1 / 2}\right) & \\
=\frac{\mathfrak{R}_{\infty}(Z \alpha)^{2}}{16}[ & {\left[1+\frac{5}{8}(Z \alpha)^{2}\right]\left(1-\frac{m}{M}\right) } \\
& \left.+\frac{\alpha}{\pi}\left(1-2 \frac{m}{M}\right)+2 \frac{\alpha}{\pi}(Z \alpha)^{2} \ln Z \alpha\right],
\end{aligned}
$$

TABLE I. Input data for the calculation of quenching anisotropies in hydrogenic ions.

\begin{tabular}{ccccc}
\hline \hline Ion & $\begin{array}{c}E\left(2 p_{3 / 2}\right)-E\left(2 p_{1 / 2}\right) \\
(\mathrm{Hz})\end{array}$ & $\begin{array}{c}\Gamma\left(2 p_{1 / 2}\right) \\
\left(\mathrm{sec}^{-1}\right)\end{array}$ & $\begin{array}{c}\Gamma\left(2 p_{3 / 2}\right) \\
\left(\mathrm{sec}^{-1}\right)\end{array}$ & $\begin{array}{c}\alpha_{\mathrm{hfs}}{ }^{\mathrm{a}} \\
(\mathrm{Hz})\end{array}$ \\
\hline${ }^{1} \mathrm{H}$ & $1.09691(10)^{\mathrm{b}}$ & $6.2683(8)$ & $6.2682(8)$ & $2.6635(8)$ \\
${ }^{2} \mathrm{H}$ & $1.09721(10)$ & $6.2683(8)$ & $6.2682(8)$ & $4.0923(7)$ \\
${ }^{4} \mathrm{He}$ & $1.75594(11)$ & $1.0030(10)$ & $1.0029(10)$ & 0 \\
${ }^{7} \mathrm{Li}$ & $8.89141(11)$ & $5.0776(10)$ & $5.0768(10)$ & $2.7960(9)$ \\
${ }^{9} \mathrm{Be}$ & $2.81081(12)$ & $1.6048(11)$ & $1.6044(11)$ & $-3.5949(9)$ \\
${ }^{11} \mathrm{~B}$ & $6.86441(12)$ & $3.9183(11)$ & $3.9165(11)$ & $1.0686(10)$ \\
${ }^{12} \mathrm{C}$ & $1.42392(13)$ & $8.1256(11)$ & $8.1201(11)$ & 0 \\
${ }^{14} \mathrm{~N}$ & $2.63912(13)$ & $1.5055(12)$ & $1.5041(12)$ & $6.6021(9)$ \\
${ }^{16} \mathrm{O}$ & $4.50444(13)$ & $2.5685(12)$ & $2.5655(12)$ & 0 \\
${ }^{19} \mathrm{~F}$ & $7.21928(13)$ & $4.1147(12)$ & $4.1085(12)$ & $1.8274(11)$ \\
${ }^{20} \mathrm{Ne}$ & $1.10101(14)$ & $6.2723(12)$ & $6.2606(12)$ & 0 \\
${ }^{23} \mathrm{Na}$ & $1.61310(14)$ & $9.1844(12)$ & $9.1637(12)$ & $9.3811(10)$ \\
${ }^{24} \mathrm{Mg}$ & $2.28635(14)$ & $1.3010(13)$ & $1.2975(13)$ & 0 \\
${ }^{27} \mathrm{Al}$ & $3.15171(14)$ & $1.7922(13)$ & $1.7866(13)$ & $1.5254(11)$ \\
${ }^{28} \mathrm{Si}$ & $4.24294(14)$ & $2.4110(13)$ & $2.4022(13)$ & 0 \\
${ }^{31} \mathrm{P}$ & $5.59668(14)$ & $3.1778(13)$ & $3.1645(13)$ & $3.6404(11)$ \\
${ }^{32} \mathrm{~S}$ & $7.25243(14)$ & $4.1146(13)$ & $4.0950(13)$ & 0 \\
\hline \hline
\end{tabular}

${ }^{a}$ Handbook of Chemistry and Physics, 52nd ed. (Chemical Rubber Co., Cleveland, 1971), p. E-57.

b The numbers in parentheses are the powers of ten by which the entries are to be multiplied. 
TABLE II. Data for obtaining the Lamb shift $S$ [Eq. (13)] from a measured value of the anisotropy $\boldsymbol{R}$.

\begin{tabular}{llcc}
\hline \hline Ion & $S_{0}(\mathrm{GHz})$ & $\boldsymbol{R}_{0}$ & $b$ \\
\hline${ }^{1} \mathrm{H}$ & $1.057867(0)$ & 0.139071 & 1.0187 \\
${ }^{2} \mathrm{H}$ & $1.059241(0)$ & 0.141165 & 1.0525 \\
${ }^{4} \mathrm{He}$ & $1.404205(1)$ & 0.117966 & 1.0352 \\
${ }^{7} \mathrm{Li}$ & $6.27375(1)$ & 0.104251 & 1.0218 \\
${ }^{9} \mathrm{Be}$ & $1.79791(2)$ & 0.094905 & 1.0207 \\
${ }^{11} \mathrm{~B}$ & $4.0457(2)$ & 0.087537 & 1.0160 \\
${ }^{12} \mathrm{C}$ & $7.8199(2)$ & 0.081723 & 1.0150 \\
${ }^{14} \mathrm{~N}$ & $1.36137(3)$ & 0.076829 & 1.0130 \\
${ }^{16} \mathrm{O}$ & $2.19621(3)$ & 0.072670 & 1.0114 \\
${ }^{19} \mathrm{~F}$ & $3.3431(3)$ & 0.069015 & 1.0088 \\
${ }^{20} \mathrm{Ne}$ & $4.8611(3)$ & 0.065876 & 1.0091 \\
${ }^{23} \mathrm{Na}$ & $6.8090(3)$ & 0.062994 & 1.0077 \\
${ }^{24} \mathrm{Mg}$ & $9.2560(3)$ & 0.060451 & 1.0074 \\
${ }^{27} \mathrm{Al}$ & $1.2265(4)$ & 0.058104 & 1.0059 \\
${ }^{28} \mathrm{Si}$ & $1.5907(4)$ & 0.056016 & 1.0061 \\
${ }^{31} \mathrm{P}$ & $2.0254(4)$ & 0.054082 & 1.0055 \\
${ }^{32} \mathrm{~S}$ & $2.5373(4)$ & 0.052299 & 1.0051 \\
\hline \hline
\end{tabular}

where $m / M$ is the electron-to-nuclear mass ratio, $\alpha$ is the fine-structure constant $1 / 137.036$, and $\mathbb{R}_{\infty}$ is the Rydberg constant. The hyperfine structure energy shifts are estimated from

$$
\Delta E_{n l j I F}=\frac{\alpha_{\mathrm{hfs}}}{n^{3}}\left(\frac{F(F+1)-j(j+1)-I(I+1)}{j(j+1)\left(l+\frac{1}{2}\right)}\right),
$$

where $\alpha_{\mathrm{hfs}}$ is the hyperfine structure coupling constant. The various input data are listed in Table I.

The Lamb shift corresponding to a measured value of the anisotropy can be obtained from the data in Table II. The dependence of the Lamb shift ${ }^{5}$ on the measured anisotropy at zero-field strength is expanded in the form

$$
S=S_{0}\left[1+b\left(R-R_{0}\right) / R_{0}\right],
$$

up to terms linear in $R-R_{0}$, where $S_{0}$ is the theoretical Lamb shift tabulated by $\mathrm{Mohr},{ }^{4}$ and $R_{0}$ is the corresponding theoretical anisotropy. The values of $S_{0}, R_{0}$, and $b$ are listed in Table II. The error introduced in $S$ by neglecting quadratic and higher terms in $R-R_{0}$ is less than $0.01 \%$ provided that $R$ differs from $R_{0}$ by less than $1 \%$. It is clear from the values of $b$ that the fractional change in $S$ from $S_{0}$ is nearly equal to the fractional deviation of $R$ from $R_{0}$. Although relativistic corrections to the transition integrals have a substantial effect on the values of $|A|^{2}$ and $\left|A^{\prime}\right|^{2}$ separately for large $Z$, their ratio, and therefore the values of $R$, are nearly unchanged. For hydrogenic sulphur $(Z=16)$, relativistic corrections increase $R$ by only $0.04 \%$.

The values of $R_{0}$ for ${ }^{1} \mathrm{H}$ and ${ }^{2} \mathrm{H}$ in Table II are slightly different from the values calculated previously ${ }^{8}$ in the limit of zero-field strength owing to the use of the revised Lamb shifts calculated recently by Mohr. ${ }^{4}$ There is a further small error in the $R_{0}$ for ${ }^{2} \mathrm{H}$ in Ref. 8 in the limit $\mathfrak{F} \rightarrow 0$ due to the use of an incorrect value for $\alpha_{\mathrm{hfs}}$. Using the corrected value $R_{0}=0.141165$ yields the experimental Lamb shift $S=1059.6 \pm 1.1 \mathrm{MHz}$ instead of the value $1058.7 \pm 1.1 \mathrm{MHz}$ given previously from the measured value $R=0.14121 \pm 0.00014$. Further experiments are in preparation to extend these measurements to the hydrogenic ions such as $\mathrm{O}^{7+}$ and $\mathrm{F}^{8+}$. There does not appear to be any fundamental difficulty which would prevent the attainment of at least the $\pm 0.1 \%$ accuracy already demonstrated in neutral hydrogen. ${ }^{8}$ This would be a substantial improvement over the accuracy of existing Lamb-shift measurements ${ }^{2,13-15}$ for the heavier hydrogenic ions.
*Research supported by the National Research Council of Canada and a NATO Travel Grant.

$\dagger$ Alfred P. Sloan Foundation Fellow.

${ }^{1}$ M. Leventhal, Nucl. Instrum. Methods 110,343 (1973) and earlier references therein.

${ }^{2}$ H. W. Kugel, M. Leventhal, D. E. Murnick, C. K. N. Patel, and O. R. Wood II, Phys. Rev. Lett. 35, 647 (1975).

${ }^{3}$ G. W. Erickson, Phys. Rev. Lett. 27, 780 (1971).

${ }^{4}$ P. J. Mohr, Phys. Rev. Lett. 34, $\overline{1050}$ (1975); and BeamFoil Spectroscopy, edited by I. A. Sellin and D. J. Pegg (Plenum, New York, 1975), Vol. 1, p. 89.

${ }^{5}$ G. W. F. Drake and R. B. Grimley, Phys. Rev. A 8,157 (1973).

${ }^{6}$ A. van Wijngaarden, G. W. F. Drake, and P. S. Farago, Phys. Rev. Lett. 33, 4 (1974).

${ }^{7}$ G. W. F. Drake and R. B. Grimley, Phys. Rev. A 11 , 1614 (1975).
${ }^{8}$ G. W. F. Drake, P. S. Farago, and A. van Wijngaarden, Phys. Rev. A 11, 1621 (1975).

${ }^{9}$ I. P. Grant, J. Phys. B 7, 1458 (1974).

${ }^{10} \mathrm{H}$. A. Bethe and E. E. Salpeter, Quantum Mechanics at One-and Two-Electron Atoms (Springer-Verlag, Berlin, 1957), p. 69.

${ }^{11}$ The dependence on $\theta$ and $\varphi$ in Eq. (8) differs from that given in Ref. 8 [Eq. (17)] only because the electric field has been arbitrarily chosen to lie along the $z$ axis instead of the $x$ axis.

${ }^{12}$ B. E. Lautrup, A. Peterman, and E. de Raphael, Phys. Rep. 3, 193 (1972).

${ }^{13}$ M. Leventhal, D. E. Murnick, and H. W. Kugel, Phys. Rev. Lett. 28, 1609 (1972).

${ }^{14}$ G. P. Lawrence, C. Y. Fan, and S. Bashkin, Phys.

Rev. Lett. 28,1612 (1972).

${ }^{15} \mathrm{H}$. W. Kugel, M. Leventhal, and D. E. Murnick, Phys. Rev. A 6, 1306 (1972). 